\title{
Noninvasive imaging in children with hypertension
}

\author{
Derek J. Roebuck • Clare A. McLaren
}

Received: 11 February 2013 /Accepted: 12 February 2013/Published online: 3 March 2013

(C) Springer-Verlag Berlin Heidelberg 2013

\section{Introduction}

High blood pressure in childhood is a significant and increasing public health problem in developed nations. The prevalence of hypertension, as defined below, is 3-4\% in American children, and at least another $3 \%$, possibly many more, fall into a borderline category known as prehypertension $[1,2]$. There are important associations between hypertension and obesity, abnormal blood lipid profiles, and diabetes mellitus. Overweight children, defined as a body mass index $>25 \mathrm{~kg}$ $\mathrm{m}^{-2}$, are more likely to have hypertension, abnormal lipids and insulin resistance than non-overweight [3]. The relationship between childhood and adult blood pressure is complex, but there is reasonable evidence that hypertension in childhood leads to adult hypertension and is associated with early markers of atherosclerosis [4]. Therefore it is a significant risk factor for the development of cardiac disease, chronic kidney disease (CKD) and stroke [5]. By extension treatment of hypertension in childhood reduces morbidity and mortality in later life.

\section{Causes of hypertension}

Primary (essential) hypertension is uncommon in preschool children, and even in older children secondary causes are found in up to $40 \%$ [6]. About $80 \%$ of secondary pediatric hypertension is caused by renal parenchymal disease, and when this is the cause the diagnosis is usually straightforward. The converse is also true, i.e. hypertension is common in children with chronic kidney disease [7] and present in

D. J. Roebuck $(\bowtie) \cdot$ C. A. McLaren

Department of Radiology, Great Ormond Street Hospital,

London, UK WC1N 3JH

e-mail: derek.roebuck@gosh.nhs.uk about half of children on hemodialysis [8] or following transplantation [9]. The next most common cause of secondary hypertension is renovascular disease. Most of the rarer causes, such as late-diagnosed coarctation or pheochromocytoma or other tumors, are suspected on clinical or laboratory grounds and confirmed with noninvasive imaging.

Children with hypertension face the possibility of a lifetime of medication, with potential adverse effects and problems related to nonadherence, particularly in adolescence. It is therefore extremely important to exclude treatable secondary causes as early as possible, and this is where pediatric radiologists have an important role.

\section{Definitions of hypertension}

Clearly not all children with a high blood pressure (BP) reading need imaging investigation, so some form of selection must be applied. First, the significance of the BP should be established. Measurements made in a clinic may overestimate (or less commonly underestimate) a child's hypertensive status. To avoid this problem 24-h ambulatory blood pressure monitoring (ABPM) is often used to provide a more comprehensive assessment. ABPM avoids the problem of overestimating hypertension caused by a "white coat effect." It also allows assessment of BP during sleep, which may be important because absence of nocturnal dipping appears to be a strong indicator of clinically significant hypertension [10].

Nevertheless, hypertension in children is defined as a BP greater than the 95th centile for age, gender and height on three different occasions $[5,11]$. (By definition, then, exactly $5 \%$ of children should be hypertensive. Why, then, are only $3-4 \%$ of children hypertensive? The reason is likely to be regression to the mean, i.e. measuring $\mathrm{BP}$ on three 
separate clinic visits decreases the probability that all will exceed the 95th centile. Another way of looking at this is that the normative values are too high. An alternative explanation is that we are getting systematically lower BP readings because of the replacement of mercury sphygmomanometers.)

Prehypertension is defined as BP between the 90th and 95th centiles (or more than $120 / 80 \mathrm{mmHg}$, even if this is below the 90th centile). Prehypertension is not normally treated with drugs unless the child has comorbidities such as diabetes mellitus, chronic kidney disease or left ventricular hypertrophy. All children with BP $>90$ th centile should be given advice about lifestyle changes, including weight loss for those who are overweight or obese (body mass index $>30 \mathrm{kgm}^{-2}$ ), instituting a healthy, low-sodium diet and getting regular physical activity. Adolescents should be advised to avoid tobacco and alcohol [12-15]. Although this seems a sensible attempt at primary prevention in children, there is actually very little evidence that it is effective in reducing $\mathrm{BP}$. The threshold for commencing drug treatment in children is stage 2 hypertension, which is defined as BP $>99$ th centile plus $5 \mathrm{mmHg}$. There is a good case that stage 2 patients should be referred to centers with special expertise in the diagnosis and treatment of pediatric hypertension $[11,16,17]$. Children with stage 1 hypertension are only treated if they have comorbidities or symptoms or fail to respond to lifestyle changes [16].

\section{Noninvasive imaging}

The main roles of noninvasive imaging are to confirm clinically suspected causes of secondary hypertension, to detect unsuspected causes where possible and to evaluate any complications $[13,18]$. Most children undergo abdominal US and echocardiography for this purpose [19]. It is possible to detect some but not all stenosis of a main renal artery using various US parameters, but the sensitivity is not adequate for detection of intrarenal lesions [20-22].

For this reason, some authors recommend a sequential increase in imaging intensity, from US through CT and MR imaging with MR angiography (MRA) to digital subtraction angiography (DSA) [19]. This strategy appears illogical to us, because significant problems can arise if we try to exclude a renovascular cause for hypertension on the basis of noninvasive imaging. One might speculate that this stepwise imaging is attractive in a fee-for-service environment, but radiologists in salaried practice often follow the same approach.

Our approach, however, is based on the argument that it is crucial to detect a renovascular cause of hypertension as soon as possible, for several reasons. First, renal artery stenosis is almost always treatable. Second, it is often progressive, sometimes rapidly so, and early detection may prevent the loss of a kidney or part of a kidney. Finally, the burden of lifelong antihypertensive medication and the risks of inadequate blood pressure control are significant and should be avoided [17].

It seems reasonable to assume that some children with elevated blood pressure and no sign of renovascular disease or coarctation on US need no further imaging. At our institution children with hypertension and no clinical, laboratory or US evidence of vascular disease whose blood pressure is controlled on one or two drugs are monitored closely without further imaging (Table 1). Conversely, in some children the probability of renovascular disease (RVD) is so high that it seems sensible to proceed to digital subtraction angiography immediately, regardless of US findings (Table 1) [23].

Whether there is an intermediate group where other noninvasive imaging would be beneficial is an open question. It is crucial to realize that the ability to demonstrate treatable renovascular disease is quite unimportant unless the sensitivity of the technique is high enough that a negative test effectively excludes this etiology. Another way of stating this is that a test that shows the presence of RVD, no matter how beautifully, is of no benefit to the child if the next step is to perform digital subtraction angiography anyway. Failure to recognize this has led to misleading claims in the literature, for example that DSA can be avoided if CT angiography (CTA) is normal [24].

Table 1 Indications for digital subtraction angiography in a child with hypertension

Findings

Clinical findings

- BP very high or difficult to control

-Systolic BP $>180 \mathrm{mmHg}$

-Failure to control BP with $>1$ drug

- Symptomatic hypertension

-Neurological symptoms or signs (including facial palsy)

-Hypertensive cardiomyopathy

- Abdominal bruit

- History of umbilical artery catheterization

- Neurofibromatosis type 1

Laboratory findings

- Elevated peripheral plasma renin

- Moderate hypokalemia

Noninvasive imaging findings suggestive of treatable vascular disease

- Direct visualization of stenosis of aorta or renal or other major artery

- Abnormal Doppler waveform in aorta and/or renal arteries

-Excluding thoracic aortic coarctation

- Delayed, diminished or absent perfusion of all or part of a kidney

- Unexplained discrepancy in renal sizes

$B P$ blood pressure 
In addition to US, three imaging modalities can, in principle, be used to detect RVD noninvasively: nuclear medicine, CT and MRI. When considering these techniques it is crucial to rely on evidence from pediatric practice because the different distribution of arterial lesions in children [25] makes evidence from research on adults essentially irrelevant.

\section{Nuclear medicine}

It is unlikely that there is any significant incremental value of dimercaptosuccinic acid (DMSA) scintigraphy over US in this context. Captopril-primed scintigraphy also appears to be insufficiently sensitive and specific, at least as it is usually performed [26]. Although it is possible that refinements in scintigraphic techniques could improve accuracy, the high frequency of bilateral and intrarenal arterial disease in children $[25,27]$ might prevent this.

\section{CT}

Recent developments have led to significant improvements in image quality in CTA [28]. In this issue of the journal, Epelman et al. [29] review the technical aspects and imaging findings in CTA performed during the evaluation of children with hypertension caused by various pathological processes. Their work is important for several reasons. Their elegant images demonstrate that at least some intraparenchymal arterial lesions and collateral vessels can be characterized with this technique. The publication by Epelman et al. [29] of CT dose parameters and sizespecific dose estimates is an important step forward. Although these do not allow direct estimation of risk to the patient, they are important if we are to compare CTA and DSA. The challenge is for angiographers to produce dose data of their own.

The images published by Epelman et al. [29] are impressive but they have not shown that CTA can replace DSA in the detection of renovascular disease. This would only be true if a study of both techniques showed that CTA missed a clinically insignificant number of patients with treatable renovascular disease. In this context a "clinically insignificant" proportion would be very low because of the high importance of detecting RVD. This is, in our view, very unlikely with current technology, because such a large proportion of lesions occur in very small arteries $[25,28]$. Although it is true that the extraparenchymal renal arteries are the most common site of disease, not all lesions in these vessels are detectable by CTA, and endovascular treatment of intraparenchymal arteries (by angioplasty or ethanol ablation) is often possible. We therefore disagree with the conclusion of Epelman et al. [29] that CTA spares children the risk of DSA [29]. In addition, it should be stressed that in centers such as ours, where the first attempt at treatment of renovascular disease is almost always endovascular, the addition of CTA to the imaging algorithm actually increases risk by adding unnecessary radiation exposure.

\section{MRI}

MR imaging and MRA have the obvious attraction that they do not necessitate exposure to ionizing radiation. They are excellent for the evaluation of large blood vessels but cannot reliably detect lesions in intrarenal or accessory arteries. It might be possible to improve the spatial resolution of MR techniques or to use an indirect sign such as delayed perfusion of an area of renal parenchyma to detect arterial lesions indirectly.

MR imaging and MRA are indicated in suspected acutephase Takayasu arteritis, because small arteries are not involved and the detection of large artery stenosis and aneurysms and arterial wall enhancement is clinically important [30]. In addition, endovascular intervention in the acute phase of Takayasu arteritis might not be advisable, which would remove most of the additional value of DSA over MR.

Another potential indication for MR is the evaluation of children with Williams syndrome, where nearly all clinically relevant lesions are in the aorta and main renal arteries. In these circumstances MR is probably preferable to CTA, and DSA is probably not routinely indicated because endovascular treatment is not usually attempted.

\section{Digital subtraction angiography (DSA)}

DSA is often referred to as the gold standard for the diagnosis of renovascular disease [17, 24, 29, 31], but this is not strictly accurate because although it is clearly the best technique for detection of renal artery stenosis, it has significant limitations [30]. These include the projectional nature of conventional DSA, which means that significant stenoses can be missed, as well as fairly poor performance in the detection of minor dissections, webs and mural thrombus. This is understandable because DSA shows the lumen of the vessel, but provides no direct information about arterial wall pathology. Some of these deficiencies can be remedied by the addition of newer techniques, including 3-D (rotational) angiography, intravascular US and optical coherence tomography. 


\section{Treatment planning}

Although we have been dismissive of the value of positive noninvasive imaging tests, it could be argued that they are important for treatment planning, for example, in directing children to endovascular or surgical treatment or for ordering equipment such as the correct size of angioplasty balloon before a DSA procedure. We do not agree with this. At our institution very few children undergo surgery without having DSA and an attempt at endovascular treatment first. Even if endovascular treatment is unsuccessful, DSA often provides useful information for planning subsequent surgery. Ideally all children should undergo endovascular treatment in a center that is equipped with a full range of angioplasty catheters, including cutting balloons, as well as stents and stent-grafts.

\section{The future}

If the number of children with essential hypertension related to overweight and obesity continues to increase, the proportion with secondary hypertension will fall. This will require refinements in our method of selection of children for imaging other than US. The most important change in clinical practice will come, perhaps in the near future, when it is possible to achieve the spatial resolution required to show almost all treatable arterial lesions with noninvasive imaging.

\section{References}

1. Hansen ML, Gunn PW, Kaelber DC (2007) Underdiagnosis of hypertension in children and adolescents. JAMA 298:874-879

2. McNiece KL, Poffenbarger TS, Turner JL et al (2007) Prevalence of hypertension and pre-hypertension among adolescents. J Pediatr 150:640-644

3. Freedman DS, Dietz WH, Srinivasan SR et al (1999) The relation of overweight to cardiovascular risk factors among children and adolescents: the Bogalusa Heart Study. Pediatrics 103:1175-1182

4. McCrindle BW (2010) Assessment and management of hypertension in children and adolescents. Nat Rev Cardiol 7:155-163

5. Falkner B (2010) Hypertension in children and adolescents: epidemiology and natural history. Pediatr Nephrol 25:1219-1224

6. Flynn J, Zhang Y, Solar-Yohay S et al (2012) Clinical and demographic characteristics of children with hypertension. Hypertension 60:1047-1054

7. Swinford RD, Portman RJ (2004) Measurement and treatment of elevated blood pressure in the pediatric patient with chronic kidney disease. Adv Chron Kidney Dis 11:143-161

8. Mitsnefes MM (2012) Cardiovascular disease in children with chronic kidney disease. J Am Soc Nephrol 23:578-585
9. Rees L (2009) Long-term outcome after renal transplantation in childhood. Pediatr Nephrol 24:475-484

10. Flynn JT, Urbina EM (2012) Pediatric ambulatory blood pressure monitoring: indications and interpretations. J Clin Hypertens (Greenwich) 14:372-382

11. National High Blood Pressure Education Program (NHBPEP) Working Group (2004) The fourth report on the diagnosis, evaluation, and treatment of high blood pressure in children and adolescents. Pediatrics 114:555-576

12. Batisky DL (2010) Obesity and the role of lifestyle and dietary intervention in the management of pediatric hypertension. J Med Liban 58:171-174

13. Brady TM, Feld LG (2009) Pediatric approach to hypertension. Semin Nephrol 29:379-388

14. Flynn JT, Falkner BE (2011) Obesity hypertension in adolescents: epidemiology, evaluation, and management. J Clin Hypertens (Greenwich) 13:323-331

15. Meyers K, Falkner B (2009) Hypertension in children and adolescents: an approach to management of complex hyper-tension in pediatric patients. Curr Hypertens Rep 11:315-322

16. Luma GB, Spiotta RT (2006) Hypertension in children and adolescents. Am Fam Physician 73:1558-1568

17. Marks SD, Tullus K (2012) Update on imaging for suspected renovascular hypertension in children and adolescents. Curr Hypertens Rep 14:591-595

18. Roebuck D (2008) Childhood hypertension: what does the radiologist contribute? Pediatr Radiol 38:S501-S507

19. Andrade H, Antonio N, Rodrigues D et al (2010) High blood pressure in the pediatric age group. Rev Port Cardiol 29:413-432

20. Brun P, Kchouk H, Mouchet B et al (1997) Value of Doppler ultrasound for the diagnosis of renal artery stenosis in children. Pediatr Nephrol 11:27-30

21. Garel L, Dubois J, Robitaille P et al (1995) Renovascular hypertension in children: curability predicted with negative intrarenal Doppler US results. Radiology 195:401-405

22. Rosendahl W, Grunert D, Schoning M (1994) Duplex sonography of renal arteries as a diagnostic tool in hypertensive children. Eur J Pediatr 153:588-593

23. Tullus K, Brennan E, Hamilton G et al (2008) Renovascular hypertension in children. Lancet 371:1453-1463

24. Vade A, Agrawal R, Lim-Dunham J et al (2002) Utility of computed tomographic renal angiogram in the management of childhood hypertension. Pediatr Nephrol 17:741-747

25. Vo NJ, Hammelman BD, Racadio JM et al (2006) Anatomic distribution of renal artery stenosis in children: implications for imaging. Pediatr Radiol 36:1032-1036

26. Abdulsamea S, Anderson P, Biassoni L et al (2010) Pre- and postcaptopril renal scintigraphy as a screening test for renovascular hypertension in children. Pediatr Nephrol 25:317-322

27. Shroff R, Roebuck DJ, Gordon I et al (2006) Angioplasty for renovascular hypertension in children: 20 -year experience. Pediatrics 118:268-275

28. Guillerman RP (2011) Newer CT applications and their alternatives: what is appropriate in children? Pediatr Radiol 2:534-548

29. Epelman M, Kurian J, Darge K et al (2013) The role of CT angiography in the evaluation of paediatric renovascular hypertension. Pediatr Radiol. doi:10.1007/s00247-012-2567-z, [Epub ahead of print] 04 Dec 2012

30. Cakar N, Yalcinkaya F, Duzova A et al (2008) Takayasu arteritis in children. J Rheumatol 35:913-919

31. Tullus K (2011) Renal artery stenosis: is angiography still the gold standard in 2011? Pediatr Nephrol 26:833-837 